\title{
THE HARD X-RAY PERSPECTIVE ON THE SOFT X-RAY EXCESS
}

\author{
Ranjan V. Vasudevan ${ }^{1}$, Richard F. Mushotzky ${ }^{1,2}$, Christopher S. Reynolds ${ }^{1,2}$, Andrew C. Fabian ${ }^{3}$, \\ Anne M. Lohfink ${ }^{1}$, Abderahmen Zoghbi ${ }^{1,2}$, Luigi C. Gallo ${ }^{4}$, And Dominic Walton ${ }^{5}$ \\ ${ }^{1}$ Department of Astronomy, University of Maryland, College Park, MD 20742-2421, USA; ranjan@ astro.umd.edu \\ 2 Joint Space-Science Institute (JSI), College Park, MD 20742-2421, USA \\ ${ }^{3}$ Institute of Astronomy, Madingley Road, Cambridge CB3 OHA, UK \\ ${ }^{4}$ Department of Astronomy and Physics, Saint Mary's University, 923 Robie Street, Halifax, Nova Scotia B3H 3C3, Canada \\ ${ }^{5}$ Cahill Centre for Astronomy and Astrophysics, California Institute of Technology, Pasadena, CA 91125, USA \\ Received 2013 November 12; accepted 2014 February 12; published 2014 March 21
}

\begin{abstract}
The X-ray spectra of many active galactic nuclei exhibit a "soft excess" below $1 \mathrm{keV}$, whose physical origin remains unclear. Diverse models have been suggested to account for it, including ionized reflection of X-rays from the inner part of the accretion disk, ionized winds/absorbers, and Comptonization. The ionized reflection model suggests a natural link between the prominence of the soft excess and the Compton reflection hump strength above $10 \mathrm{keV}$, but it has not been clear what hard X-ray signatures, if any, are expected from the other soft X-ray candidate models. Additionally, it has not been possible up until recently to obtain high-quality simultaneous measurements of both soft and hard X-ray emission necessary to distinguish these models but upcoming joint $X M M-N U S T A R$ programs provide precisely this opportunity. In this paper, we present an extensive analysis of simulations of XMM-NuSTAR observations, using two candidate soft excess models as inputs, to determine whether such campaigns can disambiguate between them by using hard and soft X-ray observations in tandem. The simulated spectra are fit with the simplest "observer's model" of a blackbody and neutral reflection to characterize the strength of the soft and hard excesses. A plot of the strength of the hard excess against the soft excess strength provides a diagnostic plot which allows the soft excess production mechanism to be determined in individual sources and samples using current state-of-the-art and next generation hard X-ray enabled observatories. This approach can be straightforwardly extended to other candidate models for the soft excess.
\end{abstract}

Key words: accretion, accretion disks - galaxies: active - X-rays: galaxies

Online-only material: color figures, supplemental data

\section{INTRODUCTION}

Matter accreting onto supermassive black holes produces emission from radio-to-X-ray energies, with a variety of different physical processes being relevant in each band. The direct luminous thermal power output from the central accretion disk appears in the optical-UV regime; a corona of electrons above the accretion disk Compton up-scatters this radiation to produce a power-law X-ray tail extending to hundreds of $\mathrm{keV}$ or greater depending on the coronal temperature. This ensemble is often shrouded by dust and gas which introduces absorption features, including a low-energy fall-off from photoelectric absorption, and troughs/edges from ionized absorption. Fall-offs are observed at higher energies due to Compton-scattering, and $\mathrm{X}$-ray reflection from the accretion disk can reprocess coronal emission to produce a distinctive set of continuum features, including a reflection hump peaking at $30 \mathrm{keV}$ and a broad Iron line at or near $6.4 \mathrm{keV}$.

Many active galactic nuclei (AGNs) exhibit an excess at soft $\mathrm{X}$-ray energies $(\lesssim 2 \mathrm{keV})$ over the coronal power law. This was first noticed by Holt et al. (1980) and Pravdo et al. (1981), and more definitely confirmed with broadband observations by Singh et al. (1985) and Arnaud et al. (1985). The feature can be modeled as a blackbody with an almost universal effective temperature of $\sim 0.1-0.2 \mathrm{keV}$. The physics of this feature remains uncertain, despite it contributing a potentially significant fraction of the total luminous power output of AGN. The small range of effective temperatures observed disfavors a scenario where it represents the hard tail of the accretion disk spectrum peaking in the far-UV (e.g., Miniutti et al. 2009; Ponti et al. 2010), as sources with different masses and accretion rates would be expected to exhibit soft excesses with different temperatures. Instead, atomic processes are often invoked to explain the soft excess, whereby a series of lines are smeared together to produce the feature. Two mechanisms can be invoked here: (1) ionized reflection with light bending, in which the series of soft X-ray lines are relativistically blurred due to being produced very close to the center of the accretion flow of a rapidly spinning black hole (Ross \& Fabian 2005; Crummy et al. 2006; Walton et al. 2013) or (2) ionized absorption, where the soft excess is not really an "excess" at all but the remnants of the true power law at low energies, with the high-energy power law being absorbed by smeared, ionized absorption with very high cloud velocities (Gierliński \& Done 2004).

Both models produce statistically acceptable fits to $X M M$ data for the same set of PG quasars (Middleton et al. 2007; Crummy et al. 2006) but both required strong relativistic smearing of either the emission or absorption features. Schurch \& Done (2008) found that this was problematic in the absorption model case due to the extreme terminal velocities required for the outflows; however, the model can still explain the data if the absorption is partially covering or clumpy. In order to naturally produce a smooth soft excess with ionized reflection, one requires strong relativistic smearing from a rapidly spinning black hole, smoothing the soft features, and light bending from a corona with variable height above the accretion disk also allows the strength of the reflected emission (and thus of the soft excess) to vary relative to the power law and even to 
dominate at times. This is also supported by the observation of a time lag between the soft excess and direct continuum in the Narrow-Line Seyfert 1 galaxy 1H 0707-495 (Fabian et al. 2009; Zoghbi et al. 2010) and in a sample of 32 AGNs (De Marco et al. 2013). Other processes can also account for the soft excess, such as Comptonization of inner-disk photons (e.g., Done et al. 2012), and most recently, magnetic reconnection has also been suggested (Zhong \& Wang 2013). It is not certain, using soft-X-ray observations alone, what process is responsible for producing the soft excess (Chevallier et al. 2006; D'Ammando et al. 2008; Laha et al. 2013). In this paper, we outline the different hard X-ray signatures that two physically motivated models are expected to produce and outline how by using relatively simple fitting methods that do not require very high signal-to-noise ratio data, one can begin to distinguish between these models using the present and future generation of X-ray observatories.

Understanding the soft excess is important for determining the true bolometric output from accretion and potentially provides a perspective on the extreme physical environments in AGNs. If light-bending and ionized reflection are important, it implies that the soft excess constitutes a real component of the ionizing luminosity from the central engine and requires rapidly spinning black holes (e.g., Walton et al. 2013); whereas if ionized absorption is more relevant in some sources, it implies that the soft "excess" belies a much larger amount of ionizing flux which we do not see due to high-energy absorption. These two scenarios can drastically change the inferred bolometric luminosity from the central engine. The implications for the bolometric luminosity from other models are less clear, highlighting the pressing need to understand how the soft excess is produced.

In the ionized reflection model for the soft excess, we expect to see an accompanying hard ( $>10 \mathrm{keV}$ ) excess, but simultaneous measurements of the soft and hard bands exist only for a few sources. Hard X-ray observatories such as RXTE and Swift/ BAT have revealed such hard excesses in many AGNs, typically a smooth feature that can be produced by reflection from either the accretion disk (ionized) or more distant neutral material (e.g., the inner edge of the putative "torus," or in general, clouds of absorbing gas surrounding the AGN). The overall strength of this "Compton hump" can be measured simply using the reflection parameter $R$ of the PEXRAV model Magdziarz \& Zdziarski (1995) available in the X-ray spectral fitting package XSPEC (Arnaud 1996). The value of $R$ in this model represents the total contribution from both distant and ionized reflection (Nandra et al. 2007; Walton et al. 2010; Rivers et al. 2013); although complex, Compton-thick absorbers may also be able to explain the hard excess (Tatum et al. 2013; Miller \& Turner 2013; Turner et al. 2009). Disentangling the components from ionized and neutral reflection (e.g., Nandra et al. 2007) is important in trying to understand the soft excess, since it can only be produced by heavily blurred ionized reflection, not reflection from neutral material. However, this requires very good signal-to-noise ratio data and is only possible for a few tens of sources currently. To add confusion, ionized absorption can also produce a rising spectrum above $\sim 7 \mathrm{keV}$ until about $20 \mathrm{keV}$, similar to that seen from a Compton reflection hump. It is only with the recent advent of NuSTAR (launched in 2012 Harrison et al. 2013), that we have real prospects to break the degeneracy between these different models, as do future hard X-ray sensitive missions such as ASTRO-H (Takahashi et al. 2012) and ASTROSAT (Paul 2013).

In this paper, we ask whether the current state-of-the-art NUSTAR mission, with good sensitivity up to $\sim 50 \mathrm{keV}$, in

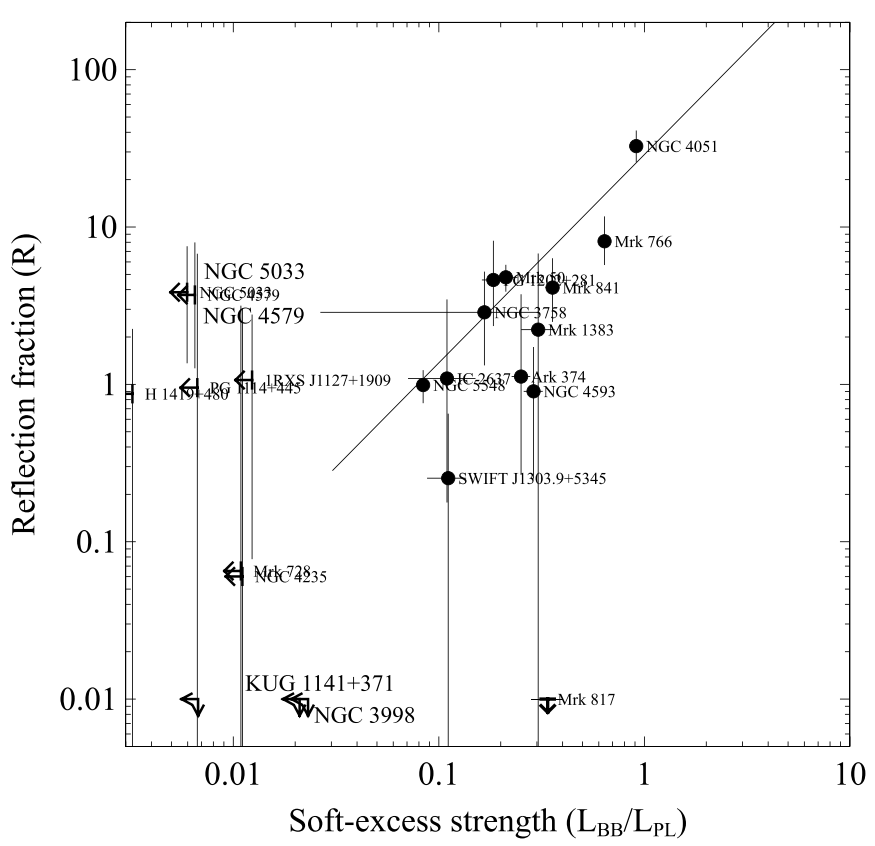

Figure 1. Reflection vs. soft excess strength measured in the Northern Galactic Cap unabsorbed $\left(\log N_{\mathrm{H}}<22\right)$ BAT AGN. The solid line shows a simple best fit to the points with well-detected soft excesses and hard X-ray reflection excesses.

combination with the excellent $0.4-10 \mathrm{keV}$ sensitivity of $X M M$, can be used to distinguish between these two scenarios using only simple characterizations of the soft and hard excess strengths, using only short ( $10 \mathrm{ks})$, inexpensive "snapshot" observations.

This paper is organized as follows. In Section 2, we describe preliminary evidence for a link between soft excess and hard excess strengths. In Section 3, we describe the simulations of this relationship using ionized reflection and absorption models. In Section 4, we discuss the results of those simulations. Finally, in Section 5, we summarize and discuss the implications of our findings.

\section{HINTS OF AN $R-S_{\text {softex }}$ RELATION FROM THE BAT AGN CATALOG}

In Vasudevan et al. (2013, V13 hereafter), we present a comprehensive analysis of the 100 Northern Galactic Cap AGN $\left(b>50^{\circ}\right)$ in the 58 month BAT catalog. The Swift/BAT instrument provides the most unbiased census of AGN due to its sensitivity in the $14-195 \mathrm{keV}$ band, less prone to the effects of absorption and host-galaxy dilution than any other band, and the Northern Galactic Cap provides a complete, manageable subsample of the catalogue from which to draw statistically robust conclusions on the AGN population. In V13, BAT and XMM data were used to constrain the reflection and soft excess strengths in 39 of these sources, including upper limits where such features were not detected. The soft excess strength is presented there as the ratio of the luminosity of the feature (using a blackbody to model it) from $0.4-3 \mathrm{keV}$, to the luminosity in a relatively "clean," feature-free portion of the primary power law from 1.5-6 keV. Below, we present a plot (Figure 1) of the reflection strength $R$ against the soft excess strength $S_{\text {softex }}=L_{\mathrm{BB}} / L_{\mathrm{PL}}$ for those 23 low-absorption sources from V13 $\left(\log N_{\mathrm{H}}<22\right)$, in which a soft excess could be detected if present. There are strong hints of a correlation 
between $R$ and soft excess strength but there are also many upper limiting reflection strengths or soft excess strengths which do not suggest any correlation. This may suggest that a different physical mechanism may account for these two families of sources. However, the results using $X M M$ and BAT need to be treated with caution: first, the BAT data are averaged over many months, whereas the $X M M$ data represent snapshots over a few tens to hundreds of kiloseconds; they are therefore not simultaneous in any way, and as is known from NGC 4051 (Ponti et al. 2006) and Mrk 590 (Rivers et al. 2012), both the measured soft excess strength and reflection can vary significantly between observations separated by a few monthsto-years. The cross-normalization between the hard and softband is therefore uncertain. For $40 \%$ of the sources in V13, the soft-band data $(0.4-10 \mathrm{keV})$ were taken during the timeframe of the BAT survey, so the BAT light curves have been used to renormalize the BAT spectra to the level appropriate for the timeframe at which the soft-band data were taken. This changes the measured reflection significantly in some sources, due to the changed relative flux at hard and soft energies. However, this only takes into account variation in the absolute normalization of the spectra, not the spectral shape, which may also vary and is only possible for the $40 \%$ of sources for which the soft-band data were taken within the time frame of the BAT survey. Therefore, using $X M M$ and BAT data together still leaves considerable uncertainties in the determination of the reflection strength $R$.

Since it is not robust to make this comparison using $X M M$ and BAT data alone, simultaneous broadband data are preferred. This is available from joint $X M M-N u S T A R$ campaigns, where the overlap between the NUSTAR and XMM bands allows the cross-normalization to be constrained very well. Such capabilities will also be offered by ASTRO-H and ASTROSAT. However, before embarking on observing programs with these missions, it is necessary to understand what the current favored models for the soft excess would predict for the relation between the soft excess strength and the measured hard excess strength. Regardless of the underlying physics responsible behind any given source spectrum, the observer's typical first-pass model will likely be a simple model such as a power law with a blackbody, and a single reflection component at high energies (modeling both distant and inner-disk reflection). Such a model has fewer degrees of freedom than complex ionized reflection or absorption models and is more readily constrained by the type of data that are typically available for most sources. In this paper, we simulate what the more complex models would look like if observed jointly by $X M M$ and $N U S T A R$ and then fit the simplest "observer's model" combination of a PEXRAV and blackbody model to measure the reflection hump and soft excess, respectively. We then investigate whether the results resemble the tentative hints of the $R$-soft excess strength relation seen in the BAT sample (Figure 1), and discuss whether XMM-NuSTAR campaigns can distinguish between different models in $R-S_{\text {softex }}$ space.

\section{SIMULATIONS}

\subsection{Ionized Reflection}

We perform all of our simulations using the XSPEC package (Arnaud 1996). The "FAKEIT" command in XSPEC provides the ability to generate a simulated spectrum for a given input model and instrumental response. We first create a grid of simulated spectra for the ionized reflection case, stepping through all available parameters over physically realistic ranges based on previ- ous studies using this model. The model combination used for generating simulated spectra is PEXRAV + KDBLUR(REFLIONX). The key component is REFLIONX (Ross \& Fabian 2005), which provides all of the essential features of the reflected continuum including the Compton reflection hump, broad iron line, and soft lines which are thought to be blurred to give rise to a soft excess. This model takes the following parameters: the iron abundance relative to solar iron abundance, $A_{\mathrm{Fe}}$ (we step this between 0.11 and 3.0, the range allowed by the model); the photon index of the illuminating coronal spectrum being reflected from the disk, $\Gamma_{\text {refl }}$ (for an input photon counts spectrum $N(E) \propto E^{-\Gamma_{\text {refl }}}$ we step this between 1.5 and 3.0, e.g., V13; Corral et al. 2011); the ionization parameter $\xi$ (the ratio of the illuminating flux to the hydrogen number density, stepped between 1 and 1000, e.g., Reynolds \& Fabian 2008) and the normalization $N_{\text {reflionx }}$. We blur this component with the KDBLUR model, which requires a radial emissivity index of the disk (we set this at 5.0 to allow for modest light-bending), the inner and outer radii of the disk ( $R_{\text {in }}$ and $R_{\text {out }}$, here frozen at $3.0 R_{\mathrm{g}}$ and $100.0 R_{\mathrm{g}}$, respectively, again to allow for relativistic effects from a spinning black hole allowing an innermost stable orbit within $6 R_{\mathrm{g}}$ ), and the inclination (which we leave at a default of $30^{\circ}$ for all realizations, assuming relatively face-on, Seyfert-1-like geometry).

The final component of the model combination to be considered is PEXRAV, which in our representation here represents the direct or illuminating power-law coronal continuum, along with simple reflection from distant, neutral material (e.g., the inner surface of the surrounding dusty gas clouds or "torus"). The PEXRAV model requires the photon index $\Gamma_{\text {direct }}$, the cut-off energy (high energy fall-off) of the spectrum $E_{\text {cut }}$, the reflection fraction $R_{\text {distant }}$, the abundances of iron and other metals $A_{\mathrm{Fe}, \text { distant }}$ and $A_{\text {other,distant }}$, and the cosine of the inclination angle and the normalization $\left(N_{\text {pexrav }}\right)$. We link the photon index of the direct component to the reflected component $\left(\Gamma_{\text {direct }}=\Gamma_{\text {refl }}\right)$ and freeze the cut-off energy at the maximal value, $10^{6} \mathrm{keV}$, since current BAT-based studies suggest that the average cut-off energy for AGN consistently lies above a few hundred $\mathrm{keV}$ in the majority of AGNs (37 out of 49 AGNs fit with PEXRAV in V13 AGN and have $E_{\text {cut }}$ values consistent with being outside the BAT band, or are poorly constrained); at any rate, we make the assumption that it lies outside the NuSTAR band, to consider the simplest case first in our simulations. We freeze the abundances at their default values (these represent the abundances of the distant reflector) and keep the inclination angle at its default value. Nandra et al. (2007) deconvolve distant and inner reflection in a sample of Seyfert AGN and, on average, find that the distant component of the reflector has a strength of $R_{\text {distant }}=0.455$ with a standard deviation of $\sigma_{\mathrm{R} \text {, dist }}=0.295$. For each realization of the input spectrum, we randomly generate a Gaussian-distributed distant reflection value using these parameters, to introduce a realistic amount of spread due to the presence of some distant reflection.

Finally, the crucial parameter in this model set-up is the ratio of the normalizations of the direct and reflected components. Before addressing this, we first need to understand a complication presented by the published version of the REFLIONX model: changing the ionization parameter actually changes the flux of the source in addition to changing the spectrum, so the normalization is not a simple flux-multiplier. We modify the table model such that the normalization is divided out from the ionization parameter and variations in $\xi$ only produce spectral shape changes (not overall flux changes). Having made this change, we can use the normalization of the REFLIONX model as a 
simple flux multiplier. We allow the ratio $N_{\text {pexrav }} / N_{\text {reflionx }}$ to go between 1.0 (representing the reflection-dominated case) and 1000.0 (where the power law dominates and ionized reflection signatures should be barely discernible in the spectrum). We finally set the overall $1-200 \mathrm{keV}$ flux of the input model to be $4 \times 10^{-11} \mathrm{erg} \mathrm{s}^{-1} \mathrm{~cm}^{-2}$, which is the measured flux for the wellknown Seyfert NGC 4051 using XMM and BAT observations, and the flux of a typical $N u S T A R$ target.

Using the above set-up, we now simulate spectra in both the XMM and NUSTAR bands. We employ more "pessimistic" assumptions for the simulated spectra and just simulate spectra for the PN instrument from XMM and the FPMA instrument from NUSTAR; if more detectors are used in data fits, then the accuracy of results obtained should only increase. For the simulated $X M M$ spectrum, we use the response and ancillary files from an observation of NGC 4051 reduced in V13, and for the $N u S T A R$ simulated spectrum, we use the latest available canned response files for the FPMA instrument. Since both the NUSTAR and XMM responses oversample the spectrum, we rebin the responses for quicker fits of the simulated spectra. This was done using the FTOOLs tasks RBNRMF and MARFRMF for rebinning the response by user-specified amounts in each group of channels and finally combining the effective area and response files. We again assume a relatively conservative exposure time of $10 \mathrm{ks}$ in both $X M M$ and $N u S T A R$, typical of the snapshots of BAT AGN currently being taken by NuSTAR; longer observations will provide better constraints on the soft and hard excess strengths. However, the key point of this study is to see whether physical models for the soft excess can be distinguished using broadband X-ray data of typical quality rather than using the longest observations available and using simple models rather than the more complex, physically motivated models. We present an example of the model spectrum and resultant simulated spectrum in Figure 2.

Having simulated the spectra, we group them both using the GRPPHA tool to have a minimum of 20 counts per bin and reload the binned spectra into XSPEC. We ignore any data outside of the range $0.4-10 \mathrm{keV}$ in the simulated $X M M$ data and any data above $80 \mathrm{keV}$ and below $3 \mathrm{keV}$ in the simulated NuSTAR data, as well as ignoring any "bad" channels. We then fit the spectra with what is henceforth referred to as the "observer's model": the simplest combination of a blackbody, a Gaussian, and a PEXRAV reflection model (BBODY + GAUSS + PEXRAV) required to account for the components seen in the spectrum. We use initial conditions that mimic a typical soft excess, power law, iron line, and hard excess spectral shape. We set the normalizations of the blackbody, Gaussian line and pexrav components to be $10^{-4}, 10^{-4}$, and $10^{-2}$, respectively, and constrain the blackbody temperature to lie between 0.01 and $2 \mathrm{keV}$, the line energy to lie between 6.3 and $7 \mathrm{keV}$, and the linewidth to lie between 0 and $0.5 \mathrm{keV}$, as is commonly done when fitting real observations. This provides a sensible starting point for the fit to ensure that the simulated spectra have a good probability of being fit successfully.

While the soft excess is probably not intrinsically blackbody emission and the hump above $10 \mathrm{keV}$ may not intrinsically be due to reflection, these model components serve to parameterize the spectral shape in a way most commonly done by observers. The purpose of the first component is to measure the strength of the soft excess when modeled as a blackbody and to calculate its strength using the simple parameterization $L_{\mathrm{BB}} / L_{\mathrm{PL}}$ introduced in V13. The purpose of the Gaussian component is to model the iron line feature that is naturally introduced around $6.4 \mathrm{keV}$ by the REFLIONX model, and the purpose of the PEXRAV component
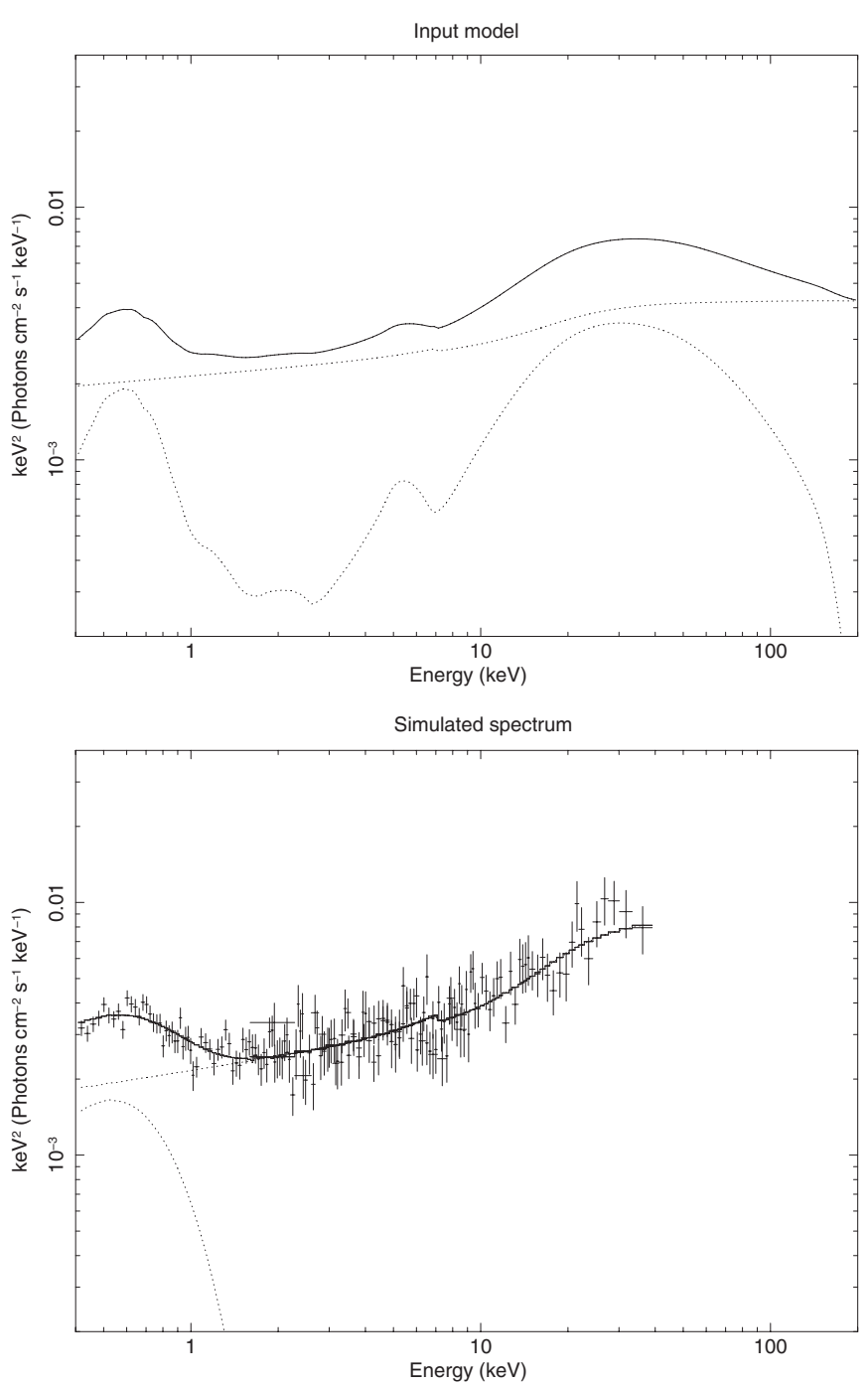

Figure 2. Model spectrum (top panel) and resultant simulated NuSTAR and $X M M$ spectra (lower panel), using ionized reflection as the input model. The simulated spectrum is fit with the simplest "observer's model" combination PEXRAV + GAUSS + BBODY. Short exposure times result in few counts above $50 \mathrm{keV}$.

is to measure the overall strength of the resultant Compton hump, which, in general, will be the sum of both distant and inner reflection or an artifact of complex absorption. For data of typical quality, the abundances and inclination are not usually uniquely determinable so we do not thaw them here for fitting and freeze them at their defaults. We also freeze the cut-off energy at the maximal $10^{6} \mathrm{keV}$ in our observer's model fit, since experience shows that this is rarely constrained to be below $\sim 200 \mathrm{keV}$ in real AGN fits using BAT and XMM data (V13). We fit each simulated spectrum with this observer's model and record all of the best-fit parameters (especially the measured soft excess and reflection strengths) for each set of input (simulated) parameters $\left(A_{\mathrm{Fe}}, \Gamma_{\text {direct }}, \xi\right.$, and the ratio of direct-to-reflected components, $\left.N_{\text {pexrav }} / N_{\text {reflionx }}\right)$.

We step each parameter within the ranges indicated above, using seven steps for four primary variables $\left(\xi, \Gamma, A_{\mathrm{Fe}}\right.$, and $N_{\text {pexrav }} / N_{\text {reflionx }}$ ), amounting to 2401 total simulated spectra. After the fits to the simulated data are complete, we then plot the "measured" soft excess strength against the reflection to investigate the presence of any relation (Figure 3 ). We filter out poor fits to the simulated spectra using a dual measurement 


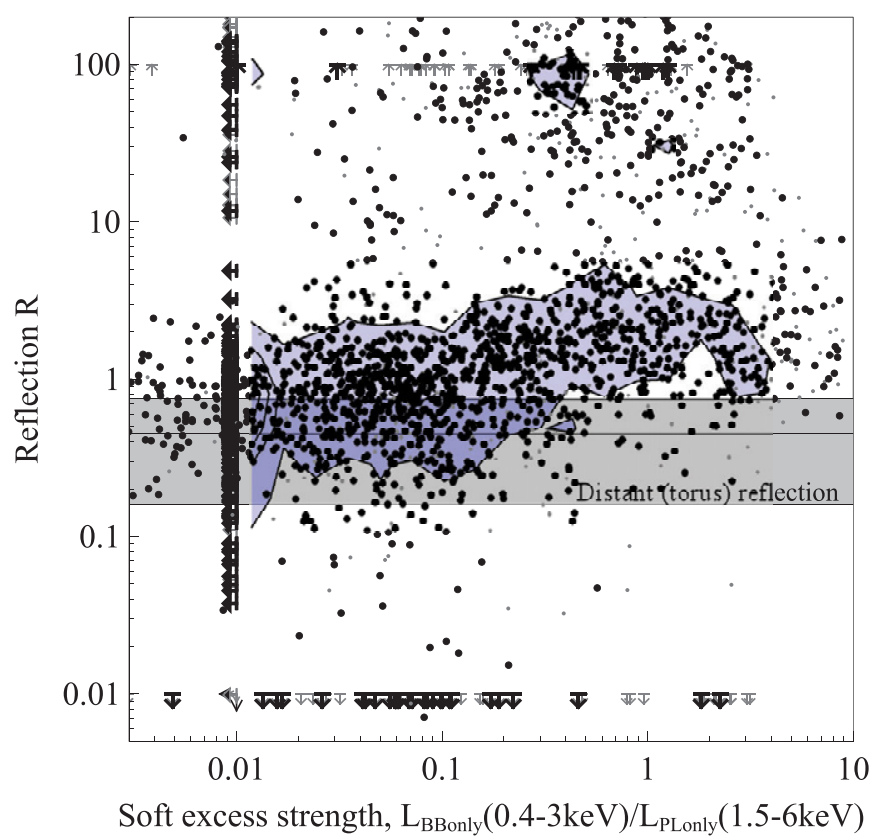

Figure 3. Reflection vs. soft excess strength as measured from ionized reflection simulated spectra. The smaller gray points show all results, and the black points show the results for which the observer's model was a "good" fit to the simulated data, using the dual- $\chi^{2}$ criteria given in the text. The contours show the clustering of the points, with the minimal contours representing $1 / 13$ th of the peak value at the center of the contours. The gray shaded area shows the expected $1 \sigma$ range of reflection strengths from distant reflection from cold material, as found by Nandra et al. (2007).

(A color version of this figure is available in the online journal.)

of the reduced $\chi^{2}$, requiring $\chi^{2} /$ dof $<6.0$ in the soft band $(<3.0 \mathrm{keV})$ and $\chi^{2} /$ dof $<2.5$ in the hard band $(>10 \mathrm{keV})$, to ensure that our observer's model fits both the soft excess and the hard excess simultaneously; a single band-wide reduced $\chi^{2}$ criterion was found to be insufficient to ensure this. These reduced $-\chi^{2}$ thresholds were chosen by visual inspection of the spectral fits for different cut-off $\chi^{2}$ values, fine-tuning the thresholds until only those spectra with visually good fits to the soft and hard excesses remained. We adopt different reduced$\chi^{2}$ thresholds in the two bands, as there are many more bins in the soft band than the hard band; it is therefore expected to be more difficult to get a good fit in the soft band using a simple two-parameter model. A total of 1962 of the 2401 fits $(82 \%)$ were deemed "good" fits according to these criteria. The "bad" fits represent parameter combinations for which the simple "observer's model" could not adequately represent the soft and hard excesses. We omit error bars and do not calculate errors on individual simulated spectrum fits but note that the error on $S_{\text {softex }}$ for typical $X M M$-quality data are very small, as shown in Figure 1. We know $N u S T A R$ will constrain $R$ much more robustly than $X M M+\mathrm{BAT}$ fits; therefore, errors on $R$ will assuredly be smaller than in Figure 1 and errors on $S_{\text {softex }}$ will be comparable.

We see a large degree of spread in Figure 3, but the contours of the highest density of points show a modest but clear trend of increasing $R$ with $S_{\text {softex }}$. Part of the region of high $R(R \gtrsim 30)$ shows a high density of points. Performing a Kendall's $\tau$ correlation analysis on the good fits only yields a correlation coefficient of 0.33 with a null-hypothesis probability $<1 \times 10^{-10}$.

\subsection{Ionized Absorption}

To illustrate the power of the $R-S_{\text {softex }}$ diagnostic plot, we also perform this exercise for the ionized wind model, SWIND1. Although Schurch \& Done (2008) find this model to require unphysically high terminal velocities of the outflow, a partially covering ionized absorber would resolve this problem, and therefore, the sWIND1 model can still be taken as representative of an important class of multiple-absorber, partially covering, ionized absorber models that can account for the soft excess. We therefore simulate spectra using this model, using the range of parameters identified in the Middleton et al. (2007) study on PG quasars, to see whether such a model can produce soft "excesses" and hard "excesses" that can be modeled as a "blackbody plus reflection" model combination.

We use the model combination SWIND1(PEXRAV) to simulate spectra, where the PEXRAV component represents the primary X-ray continuum along with some distant reflection. In Middleton et al. (2007), a more complex model for the primary X-ray power law and distant reflection is used but the salient features of such a model are reproduced here by PEXRAV for our purposes. We follow exactly the same rationale for randomly seeding the distant reflection with values appropriate for the distribution found in Nandra et al. (2007) and follow identical rationale to that given above in Section 3.1 for determining the primary continuum parameters; the primary continuum is common to both the ionized reflection and absorption cases. For the ionized absorption component, we step the column density of the wind between 3 and $50 \times 10^{22} \mathrm{~cm}^{-2}$; the photon index of the primary continuum (PEXRAV) $\Gamma$ is varied between 1.5 and 3 ; the logarithm of the ionization parameter $\xi$ is varied between $2.1<\log (\xi)<4.0$; and the Gaussian velocity dispersion of the wind is varied between 0.1 and 0.5 , all based on the parameters found from fitting data on real AGN in Middleton et al. (2007). We simulate spectra in a grid using seven steps between the maxima and minima for each parameter (see Figure 4 for an example of simulated spectra) and plot the resulting soft excess and reflection strengths in Figure 5. For this input model, 2073 out of 2401 potential parameter combinations yield successful "observer's Model" fits $(86 \%)$; in the remainder of cases, a "blackbody plus reflection" model combination entirely failed to fit the simulated spectrum (i.e., XSPEC could not compute a fit at all to produce a fit statistic). Using the same dual reduced- $\chi^{2}$ criteria as for ionized reflection, we find that 1595 of the successful fits were "good" fits (i.e., $77 \%$ of successful fits, or $66 \%$ of the total 2401 simulated spectra).

We see a large degree of spread in Figure 5, without any clear trend linking $R$ with $S_{\text {softex }}$. Notably, a large number of simulated spectra show prominent soft excesses with negligible $R$. Performing a Kendall's $\tau$ correlation analysis on the good fits only yields a correlation coefficient of -0.003 with a nullhypothesis probability of 0.88 , indicating that there is a high chance of the two properties being completely uncorrelated.

\section{DISCUSSION OF RESULTS}

At the outset, we note that the "Observer's Model" produces a successful fit to all of the simulated spectra using ionized reflection as the input model, but only $86 \%$ of the ionized absorption simulated spectra could be fit with this model. This indicates that while ionized absorption can produce a wide range of spectral shapes that can mimic the appearance of a soft blackbody component with a hard X-ray reflection hump, a substantial minority do not. Sources in those ranges of parameter 

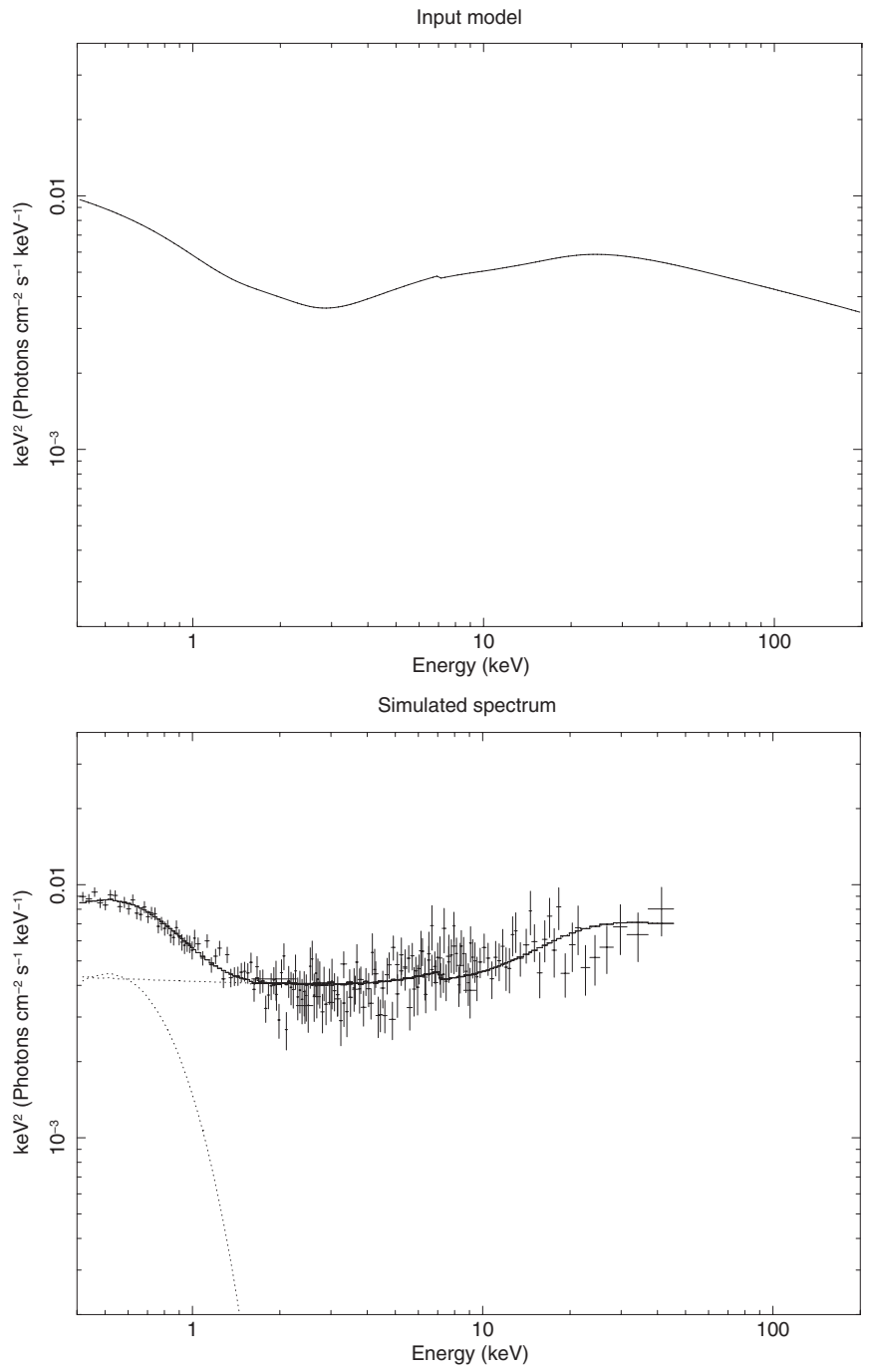

Figure 4. Model spectrum (top panel) and resultant simulated NUSTAR and $X M M$ spectra (lower panel), using ionized absorption as the input model. The simulated spectrum is fit with the "observer's model" combination PEXRAV + ввоDY. Short exposure times result in few counts above $50 \mathrm{keV}$.

space would exhibit spectral shapes clearly distinguishable from ionized reflection. We discuss this class of spectra further in Section 4.2.

The two models compared here show some clearly distinct behavior in $R-S_{\text {softex }}$ space; the results for both models are plotted in Figure 6. Notably, in the ionized reflection scenario, stronger soft excesses can be produced, and these are accompanied by stronger measured reflection fractions, particularly for $S_{\text {softex }} \gtrsim 1, R \gtrsim 1$. Ionized reflection as a mechanism for the soft excess could be distinguishable from distant reflection by a more pronounced hard excess (values of $R \gtrsim 2$ ) that one expects to obtain from such a physical process, coupled with a trend towards higher $R$ at higher $S_{\text {softex }}$, which one would expect to observe in large samples of AGNs. This would imply that in sources where both (1) strong soft excesses and (2) strong reflection are measured, ionized reflection is the most likely candidate model. For sources with $0.5 \lesssim R \lesssim 3$ and $S_{\text {softex }} \lesssim 1$, the two models are not immediately distinguishable.

Ionized absorption, on the other hand, can produce hard excesses with strengths $R$ that are not easily distinguishable from distant (torus) reflection, even if a strong soft excess is present. This would imply that for sources with (1) a strong soft

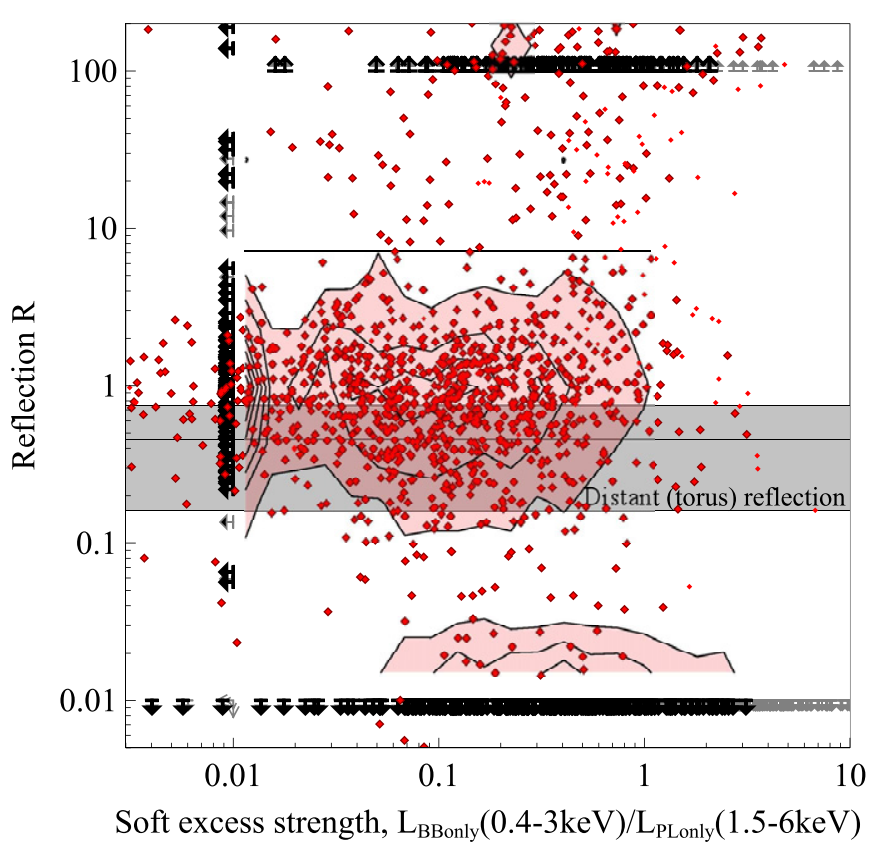

Figure 5. Reflection vs. soft excess strength as measured from ionized absorption simulated spectra. The key is as in Figure 3.

(A color version of this figure is available in the online journal.)

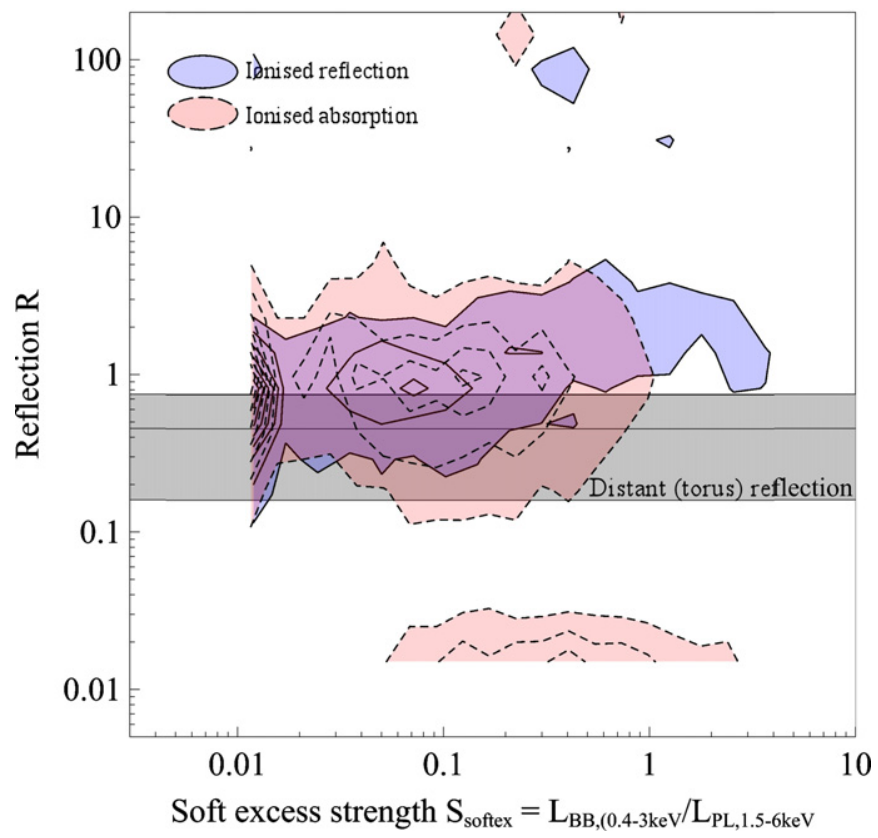

Figure 6. Contours of reflection vs. soft excess strength from both ionized reflection and ionized absorption models, for comparison.

(A color version of this figure is available in the online journal.)

excess $\left(0.3<S_{\text {softex }}<1\right)$ but (2) a not particularly strong hard excess $(R \lesssim 1)$, ionized absorption may be a candidate model to explain the soft excess, but it could also be due to an altogether different physical process with an unrelated hard excess due to distant, neutral reflection.

Chevallier et al. (2006) found that the strongest soft excesses can be produced by ionized absorption, not reflection, contrary to our findings here. They investigated the different absorber conditions required to reproduce observed soft excesses in detail, alongside a blurred reflection model akin to the one we use here but with one key difference: the reflection spectrum 
strength is constrained to be sufficiently lower than that of the primary continuum such that prominent soft excesses cannot be seen. In our model, the ratio of the direct PEXRAV continuum to the REFLIONX continuum is widely variable to account for light-bending effects (e.g., the coronal height varying above the accretion disk), and when $N_{\text {pexrav }} / N_{\text {reflionx }} \sim 1$, strong soft excesses can be observed, as seen in reflection-dominated epochs of sources such as NGC 4051 (Ponti et al. 2006). Full consideration of the effects of light-bending at the inner parts of the accretion flow allows the reflection spectrum to dominate and produce such strong excesses.

Some very interesting behavior is seen at the extrema of Figure 6. Both ionized reflection and absorption allow for nondetected soft excesses alongside moderate reflection $(0.2<$ $R<3.0$ ) but ionized absorption allows for a large range of soft excess strengths alongside negligible or no measured hard excess. Therefore, in sources where the soft excess is prominent but reflection is undetectable, it may be appropriate to consider partially covering ionized absorption or other nonreflection based models (e.g., Comptonization). Finally, the region of the plot showing strong soft excesses $S_{\text {softex }}>1$ and relatively weak hard excesses $R<1$ is not populated by either of the two models considered here. Further work needs to be done to explore if other models can occupy this part of parameter space.

We can apply these diagnostics now to the objects in Figure 1, although with the caveat that those results were obtained using nonsimultaneous hard and soft X-ray data, without the overlap between the soft and hard band required to constrain reflection well. Nevertheless, one can suggest (subject to further investigation with better data) that those objects in which reflection and soft excess strength seem to be increasing together likely have a strong contribution from ionized reflection. NGC 4051 has been studied in depth, and it is known that ionized reflection can be fit to the detailed $0.4-10 \mathrm{keV}$ spectrum (Ponti et al. 2006; Alston et al. 2013). Reflection has also been suggested for Mrk 766 (Emmanoulopoulos et al. 2011) but complex, multilayered absorbers (perhaps a disk wind) and occultation by absorbing clouds have also been invoked (Turner et al. 2007; Risaliti et al. 2011). Both ionized reflection and absorption can explain the spectrum of Mrk 841 (Cerruti et al. 2011). The object Mrk 817, which has negligible reflection but a measurable soft excess, may well require a different model such as ionized absorption to account for its soft features. Indeed, Winter et al. (2011) mention an epoch in this source where absorbing winds were detected in UV spectroscopy of this source from 1997 and 2009, albeit without the X-ray edges due to oxygen expected at 0.73 and $0.87 \mathrm{keV}$ from such outflows. One potential explanation may be that the $>2 \mathrm{keV}$ X-ray continuum in this source is absorbed by a highly relativistic ionized absorber, which may tally with the absorption signatures in the UV. Again, data that extends into the $>10 \mathrm{keV}$ band would provide more definitive answers, stressing the utility of the approach outlined in this paper.

Two Narrow-Line Seyfert 1 nuclei with strong soft excesses have recently been studied in detail using XMM data: $1 \mathrm{H} 0707$ 495 and IRAS 13224-3809. Fabian et al. (2012) and Dauser et al. (2012) find that reflection can successfully fit the spectrum of 1H 0707-495; Kara et al. (2013) and Fabian et al. (2013) find the same for IRAS 13224-3809. We use the archival XMM data to estimate $S_{\text {softex }}$ for both of these sources and find that they are 3.3 (1H 0707-495) and 2.6 (IRAS 13224-3809); therefore, according to the scheme found in this paper, their soft excesses are both sufficiently strong to favor a reflection-dominated scenario. Broadband observations with $N U S T A R$ should be able to confirm this and locate both objects on the $R-S_{\text {softex }}$ plot.

\subsection{Extreme Values of the Hard Excess Strength, $R \gtrsim 100$}

For both models tested in this paper, there are a cluster of points at $R \gtrsim 100$, which so far have not been observed in the real AGN population. For the ionized reflection case, further investigation reveals that all of the simulated spectra that produce $R>100$ have $N_{\text {pexrav }} / N_{\text {reflionx }}<10$, and all of the very extreme $R$ values (i.e., $R>200$ ) measured from "good" fits have $N_{\text {pexrav }} / N_{\text {reflionx }}=1$, the lowest value of the ratio included in the simulations corresponding to the most reflectiondominated case (see Figure 7, top panel). This suggests that for reflection-dominated spectra, the spectral shapes produced can genuinely give rise to very high, "anomalous" $R$ values (under the fitting assumptions adopted in this study), depending on the spread of other intrinsic parameters, i.e., $A_{\mathrm{Fe}}, \xi$ and $\Gamma$. We then focus only on the subset of objects with reflectiondominated spectra $\left(1<N_{\text {pexrav }} / N_{\text {reflionx }}<10\right.$, Figure 7 , lower panel). We split the results into different bins of $A_{\mathrm{Fe}}, \xi$, and $\Gamma$, selecting three bins for each (using logarithmic spacing for $\xi$ ). We find that the ionization parameter $\xi$ produces the greatest variation in measured $R$. The lowest ionization parameters, $1.0<\xi<10.0$, give rise to almost all of the $R>100$ points. In conclusion, the most reflection-dominated spectra $\left(1.0<N_{\text {pexrav }} / N_{\text {reflionx }}<10.0\right)$ coupled with the most weakly ionized reflectors $(1.0<\xi<10.0)$ can produce very strong hard excesses.

This range of $R$ values has not been seen in the real AGN population so far but only a handful of observations currently exist of reflection-dominated states of AGNs (e.g., Zoghbi et al. 2008; Fabian et al. 2012). Such prominent hard excesses may be found with new NuSTAR observations. Since the ionization parameter is proportional to the luminosity, the very low luminosity sources (with potential for the most prominent hard excesses) may also be selected out of most X-ray surveys.

For the ionized absorption model, the highest values of the measured $R$ occur for $\log (\xi)<2.75$. From inspection of the spectra, we find that many of these low ionization absorbers have absorption troughs that look more like extended "edges." The hard portion of the spectrum can then be fit as a pure reflection component in some cases, leading to high values of $R$. In the remainder of low ionization absorber spectra, the slope of the intermediate $(3-10 \mathrm{keV})$ region is too extreme to be fit by the PEXRAV component and the fit fails altogether (discussed further in Section 4.2).

We also consider the possibility that the low exposure time (10 ks) and resulting poor-quality data at high energies could lead to such anomalous $R$ values. However, increasing the observation time to $20 \mathrm{ks}$ (for both ionized reflection and absorption models, Figure 8) did not produce any significant change in the results and the broad trends seen.

\subsection{Failed Fits with Ionized Absorption}

As mentioned in Section 3.2, 14\% of the simulated ionized absorption spectra result cannot be fit with the observer's model. We investigate this class of objects in more detail. We find that the only weak discriminant of whether a spectrum fits successfully or not is the ionization parameter of the absorber, $\log (\xi)$. Failed fits only occur for spectra simulated with $2.1<\log (\xi)<2.75$. There are 1029 possible parameter combinations/simulated spectra in this range (i.e., three bins in 


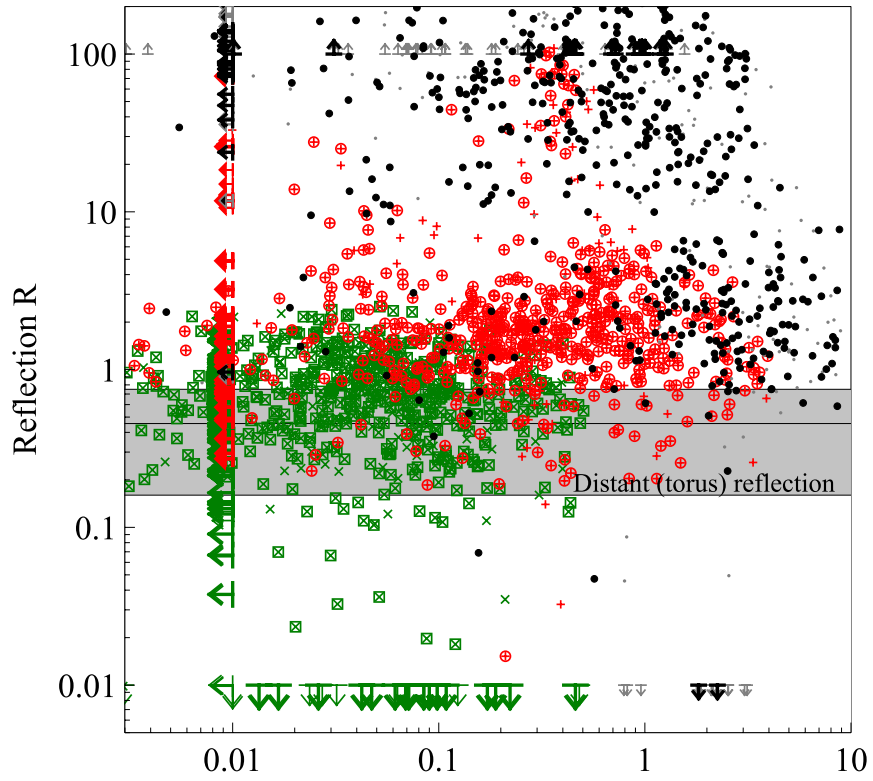

Soft excess strength, $\mathrm{L}_{\text {BBonly }}(0.4-3 \mathrm{keV}) / \mathrm{L}_{\text {PLonly }}(1.5-6 \mathrm{keV})$

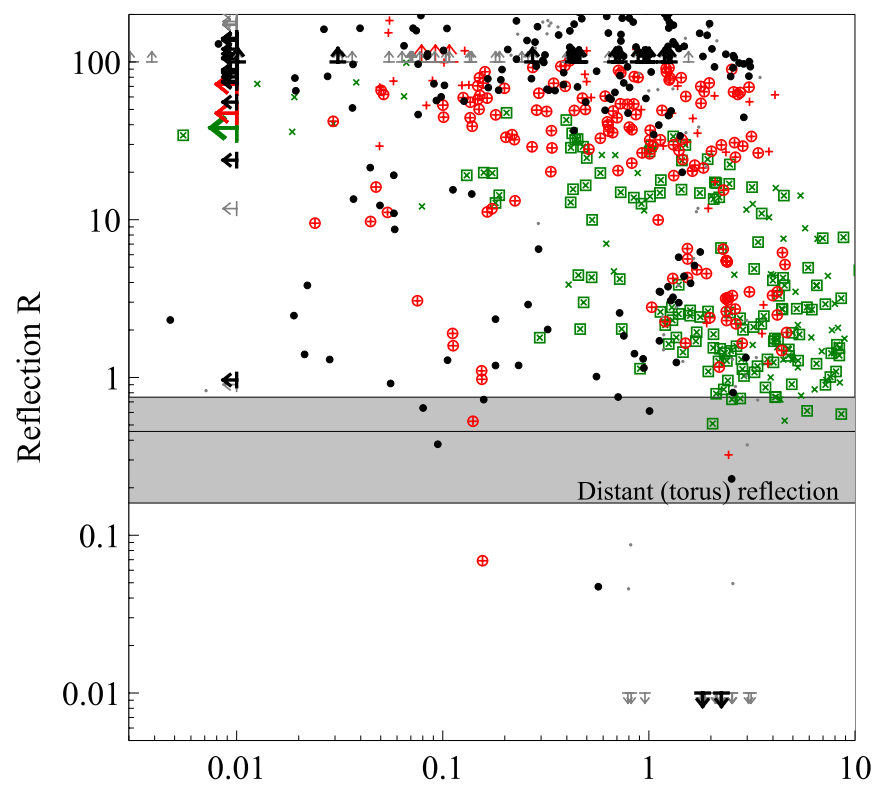

Soft excess strength, $\mathrm{L}_{\text {BBonly }}(0.4-3 \mathrm{keV}) / \mathrm{L}_{\text {PLonly }}(1.5-6 \mathrm{keV})$

Figure 7. Measured hard excess and soft excess strength for the ionized reflection scenario, split by (1) ratio of the direct-to-reflected component $N_{\text {pexrav }} / N_{\text {reflionx }}$ (top panel), and (2) the ionization parameter $\xi$ (lower panel). In the top panel, small and large black circles represent simulated spectra with $1<N_{\text {pexrav }} / N_{\text {reflionx }}<10$ (successful fits and "good" fits, respectively); red crosses (successful fits) and red circled crosses (good fits) represent $10<$ $N_{\text {pexrav }} / N_{\text {reflionx }}<100$ spectra; and green X symbols (successful fits) and green $\mathrm{X}$ symbols within squares (good fits) represent $100<N_{\text {pexrav }} / N_{\text {reflionx }}<1000$ spectra. In the lower panel, the same sequence of symbol combinations is used to split the reflection-dominated $\left(1<N_{\text {pexrav }} / N_{\text {reflionx }}<10\right)$ subset of spectra into groups with $1<\xi<10,10<\xi<100$, and $100<\xi<1000$, respectively The colors of the upper/lower limit arrows match the colors of the points for the corresponding parameter groups, and the size of the upper/lower limit symbols increases going from small to high values of $N_{\text {pexrav }} / N_{\text {reflionx }}$ and $\xi$.

(A color version of this figure is available in the online journal.)

$\log (\xi)$ and seven bins for each other parameter), out of which 328 model combinations fail completely. On inspection, these spectra exhibit ionized absorber troughs that look more like edges with a very steep rise in flux towards higher energies.

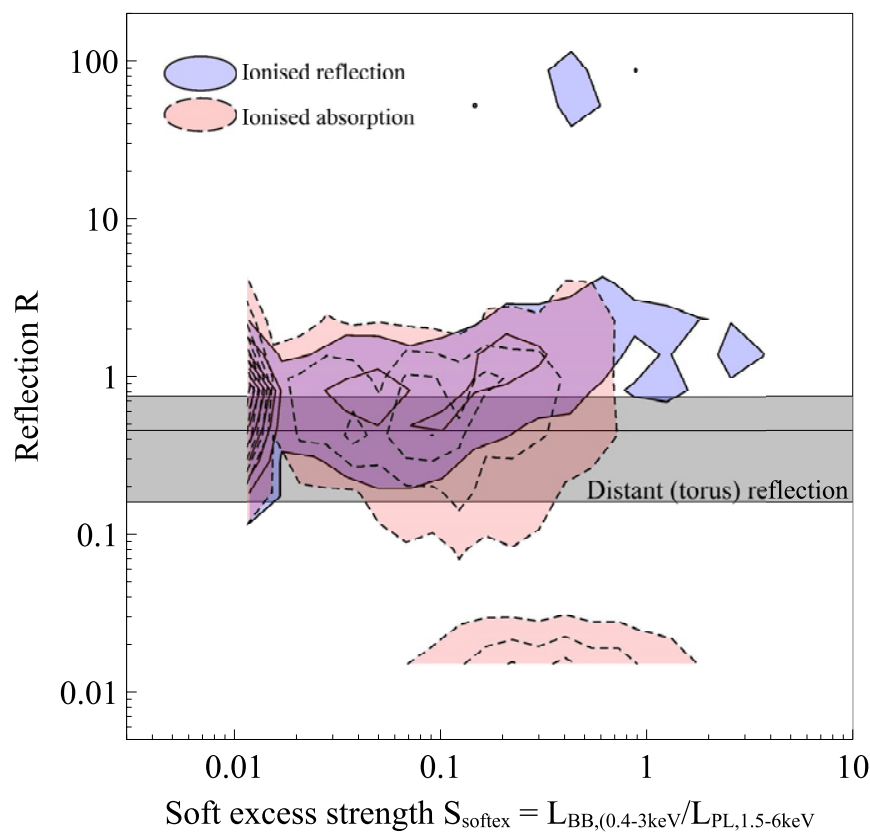

Figure 8. Simulation results for the $R-S$ diagram with an observation time of $20 \mathrm{ks}$. The key is as in Figure 3.

(A color version of this figure is available in the online journal.)

When manually fit with the observer's model combination, we find that the fits consistently fail. The power law regime of the PEXRAV component attempts to fit to the rising part of the edge below $10 \mathrm{keV}$ but the photon index required is too extreme (see Figure 9). This would be a readily identifiable subset of spectral shapes which cannot be fit with a simple blackbody and reflection model, and any soft "excess" seen would clearly be distinguishable from one produced by ionized reflection.

Of the remaining 701 low $\log \xi$ spectra which are successfully fit by the observer's model, only $50 \%$ qualify as "good fits" according to the dual- $\chi^{2}$ criteria. Inspection of these "good fits" reveals that the power-law component is able to fit the rising 1-6 keV portion of the spectrum successfully. It is therefore not straightforward to identify a very specific part of ionized absorber parameter space that eludes fitting with the observer's model but we can say that the failing of the fit is restricted to low ionization absorbers. The success rate of fitting such unusual spectral shapes may also be subtly dependent on the initial conditions employed in the observer's model, the exploration of which is beyond the scope of this paper.

\subsection{Predictions for Other Comparable Instruments}

We have presented results for the $X M M-N u S T A R$ instrument combination since NUSTAR is newly launched and this instrument combination is already being used to obtain simultaneous broadband X-ray data on AGNs (e.g., Risaliti et al. 2013; Matt et al. 2014); therefore, it is the most relevant prediction for the current circumstances with real near-term prospects of verifying the work in this paper. ASTRO-H and ASTROSAT are also on the horizon, and to check whether our predictions hold for other instruments, we also perform simulations for the ionized reflection model using the current $A S T R O-H$ predicted response matrices, assuming the same $10 \mathrm{ks}$ exposure time. We caution that these responses may be more uncertain than the XMM-NuSTAR responses, as real responses are likely to differ significantly from the prelaunch predicted ones. The resulting $R-S$ diagram does not show any significant difference to that obtained for 

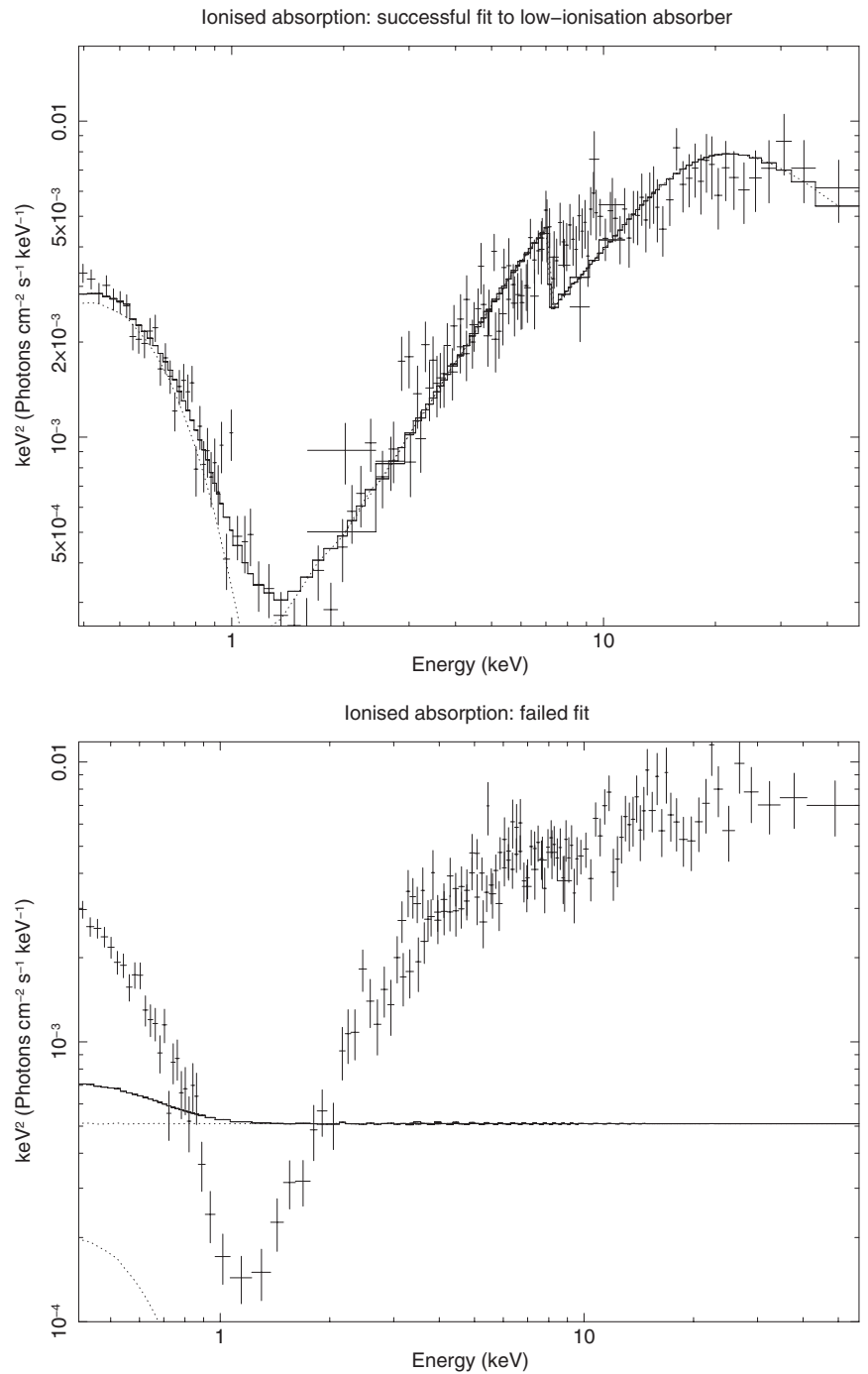

Figure 9. Example spectra with low ionization ionized absorbers, showing the attempted observer's model fit using the PEXRAV + BBODY model combination. Top panel: example of a successful fit. The input parameters for the simulated spectrum are $\Gamma=2.0, N_{\mathrm{H}}=5.0 \times 10^{23} \mathrm{~cm}^{-2}, \log \xi=2.73$, and $\sigma=0.43$. Lower panel: example of a failed fit. The input parameters are $\Gamma=2.0$, $N_{\mathrm{H}}=1.87 \times 10^{23} \mathrm{~cm}^{-2}, \log \xi=2.42$, and $\sigma=0.23$.

$X M M-N u S T A R$ (Figure 10), suggesting that the conclusions in this paper should hold for other similarly equipped future broadband X-ray observatories.

\subsection{On the Feasibility of Observing These Trends in Real AGN Samples}

The simulations here outline the trends expected in $R-S_{\text {softex }}$ space for two different models using a large grid of 2401 parameter combinations. AGN samples are typically much smaller due to the competition for observation time, so we require a measure of how feasible it will be to detect these trends in real samples of AGNs. We perform further simulations to estimate the typical AGN sample size required before a correlation (or the absence of one) can be seen between $R$ and $S_{\text {softex }}$.

We use a Monte-Carlo rejection method to simulate $N$ values of $R$ and $S_{\text {softex }}$ (corresponding to a sample of $N$ AGN) randomly determined throughout the available $R-S_{\text {softex }}$ space, using the contours determined from Figures 3 and 5 as the probability distribution with which to distribute the points in $R-S_{\text {softex }}$ space.

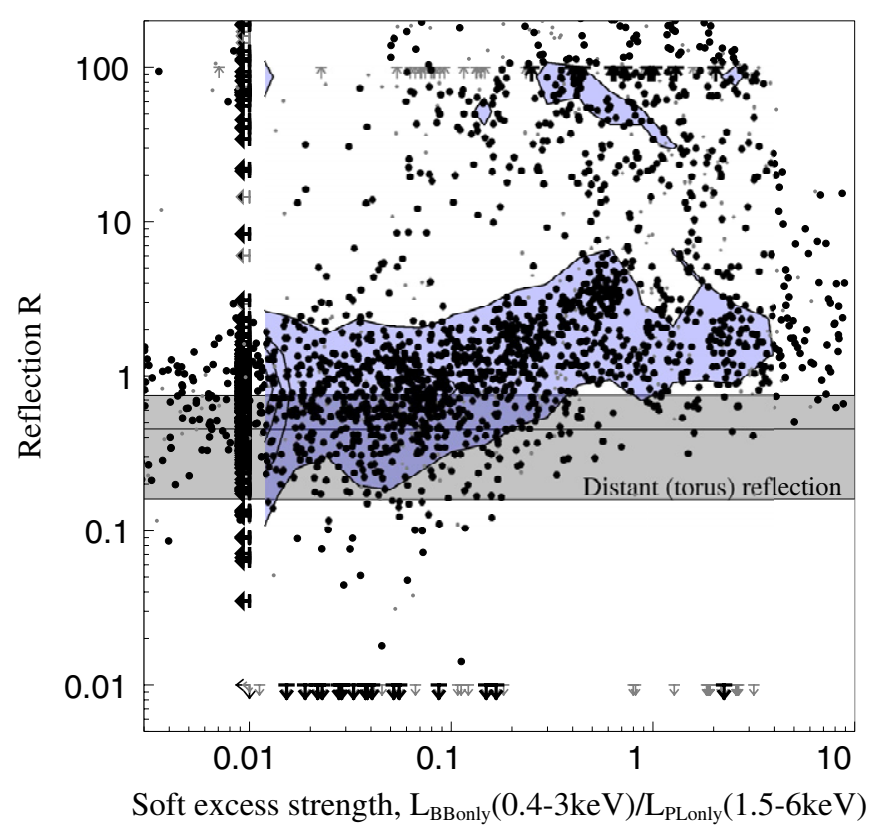

Figure 10. Results for ionized reflection using the ASTRO- $H$ response matrices. The key is as in Figure 3.

(A color version of this figure is available in the online journal.)

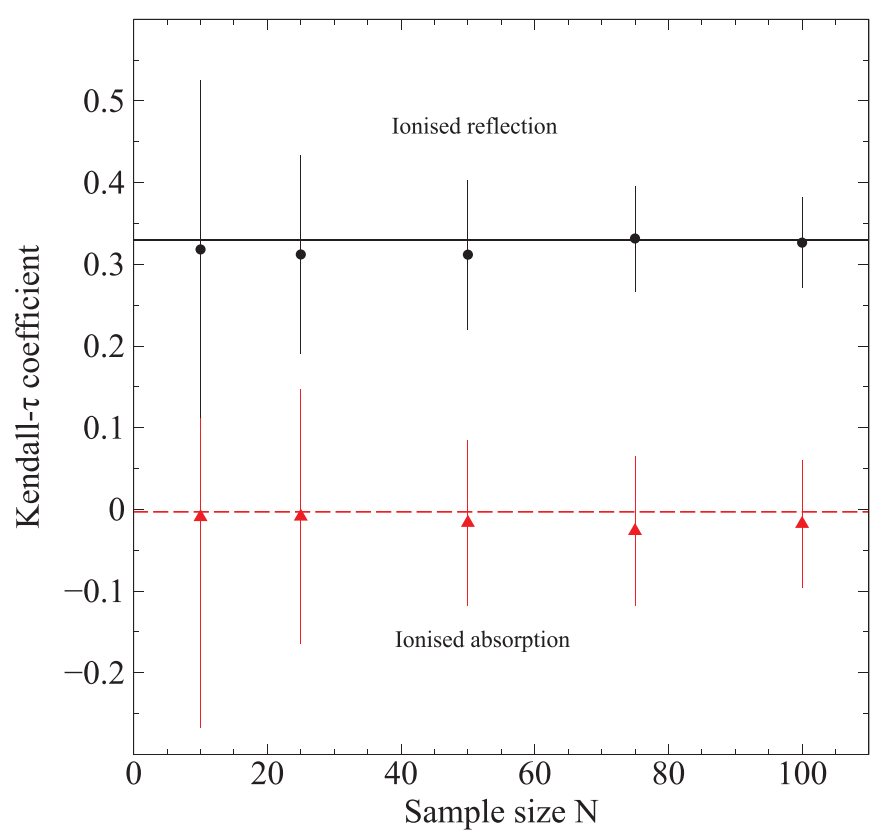

Figure 11. Kendall's $\tau$ correlation coefficient between $R$ and $S_{\text {softex }}$ against sample size, for a simulated AGN sample of size $N$. The solid (black) and dashed (red) lines show the correlation coefficients measured for the whole set of simulations (corresponding to $N>1500$ ) for ionized reflection and ionized absorption, respectively. The error bar shows the standard deviation from 100 Monte-Carlo realizations of such samples of $N$ objects and indicates that as $N$ approaches $\sim 60$, the correlations exhibited by samples of predominantly reflection or absorption dominated samples can be clearly distinguished.

(A color version of this figure is available in the online journal.)

We vary $N$ and measure the resultant Kendall's $\tau$ correlation coefficient of the faked sample to determine when the strength of the correlation approaches that seen in the full simulations. We do this for both ionized reflection and ionized absorption. The variation of the correlation coefficient with $N$ for each input model is shown in Figure 11. 
We see that at $N \gtrsim 60$, the uncertainty in the correlation coefficients produced by the two processes drops such that the two processes can be distinguished at a $2 \sigma-3 \sigma$ level. Therefore, even though the physical process may be ambiguous for an individual object based on its location in the $R-S_{\text {softex }}$ plane, the trend exhibited by a well-selected, representative AGN sample in this plane can provide an indication of the physics most relevant for the majority of AGNs in the sample.

\subsection{Outstanding Issues}

We outline some issues to be further explored in future work. First, the ratio $N_{\text {pexrav }} / N_{\text {reflionx }}$ is currently used as an estimator of the degree of light bending in the reflection scenario. A more physical understanding of this parameter is needed to place appropriate upper and lower limits on it for the simulations. It is easy to conceive of a situation where the direct power law dominates; however, understanding the lower limit on the range of physically viable ratios (here assumed to be unity) is more complex and requires a more complete consideration of the energetics of the reflection dominated state than is undertaken here. This may impact the strongest observable strengths of the soft excess from the reflection scenario, as initially investigated by Chevallier et al. (2006), but the observation of AGN in fully reflection-dominated states does support the possibility that $N_{\text {pexrav }} / N_{\text {reflionx }}$ can take very low values (even $<1$ ).

We have assumed uniform or log-uniform distributions for the input parameters in these simulations as the simplest possible scenario. However, it may be the case that the real underlying distributions are not uniform or that there are correlations between the input parameters. There are a handful of studies on this in the literature, presenting the photon index and luminosity distributions in AGNs (e.g., Corral et al. 2011; Vasudevan et al. 2013). Previous works (e.g., Reynolds \& Fabian 2008; Ballantyne et al. 2011) have found a range of ionization parameters for ionized reflection consistent with that assumed here. There are suggestions of a weak correlation between photon index and luminosity (e.g., Saez et al. 2008), and hence potential for a correlation between $\Gamma$ and $\xi$. However, there is no detailed work on the true underlying distributions of physical parameters of reflectors. It is possible that the observed trends highlighted in this work in the $R-S$ plane could be changed and the density of points in different parts of the plot could be altered, if different underlying distributions are used. To allow for this to be investigated in the future, we make our simulation results public with the online data accompanying this paper. The interested researcher can then draw from the provided results in a nonuniform way using more updated distributions or correlations between parameters, if and when such details become more well constrained. However, one instinctively expects some degree of correlation between hard and soft excess strengths in the ionized reflection scenario, regardless of the precise underlying parameter distributions.

One of the latest models to be proposed for the soft excess is the OPTXAGNF model (Done et al. 2012). Their model combines disk emission, Comptonization, and a power law in an energetically self-consistent way, where the inner part of the accretion flow below a coronal radius is Comptonized to produce the soft excess. Comptonization is the engine behind the soft excess in this model, and one does not expect it to exert any influence on the hard X-ray emission based on simple models (e.g., Page et al. 2004) providing a purely standalone component for the soft excess. Therefore, one does not expect any link between the soft excess and hard excess in this scenario.
However, this may not be the case for OPTXAGNF, where the disk and corona geometries are linked by energetic considerations, and we are currently undertaking a study of this model in detail, with a view to produce a similar $R-S_{\text {softex }}$ diagnostic plot to compare it with the models in this paper. The OPTXAGNF model is able to fully reproduce the optical-to-X-ray SED up to $10 \mathrm{keV}$, as shown by the comprehensive study of Jin et al. (2012), but its hard X-ray signatures have not been studied. Additionally, distant reflection needs to be added in a consistent fashion if we want to compare it with the models studied here, where PEXRAV was used to provide the direct continuum along with the distant reflection. How to do this is not clear, and since OPTXAGNF has many more model parameters to consider than the models in this paper, we defer this study to a future paper (in preparation).

\section{SUMMARY}

This work points to a scheme whereby different candidate models for the soft excess can be distinguished in a plot of measured reflection against soft excess strength, assuming the simplest possible PEXRAV + BBODY model combination is fit to the spectra, according to the scheme in Figure 6. This methodology can readily be extended to other candidate models, and we are currently in the process of producing such a diagnostic for the OPTXAGNF model, where photons from the inner part of the accretion disk are Comptonized to produce the soft excess (Done et al. 2012). A key advantage of this approach is its economy: data of moderate quality can be used, gathered using short exposures (e.g., $10 \mathrm{ks}$ in both $X M M$ and NuSTAR for a source of the brightness of NGC 4051) to gain real physical insight into the energy production mechanisms in AGNs, without requiring fitting of more complex models to long-exposure, very high signal-to-noise ratio data. This approach will therefore be useful in constraining the soft excess mechanism in samples of AGNs where it may be challenging to obtain such long exposures on each source. This approach is particularly useful for samples of AGNs where trends can be discerned, although it can be used for individual AGN as well, if they lie in unambiguous regions of $R-S_{\text {softex }}$ space. Notably, ionized reflection predicts a clear relation between $R$ and $S_{\text {softex }}$ but ionized absorption does not.

As simultaneous or broadband X-ray data comes in from $N U S T A R+X M M, A S T R O-H$, and ASTROSAT, this plot can be populated with accurate, simultaneous determinations of the strengths of the hard and soft excesses in samples of real AGN, updating the work presented in Figure 1. Using the contours presented in Figure 6 as probability contours, we simulate a smaller sample of AGNs using a Monte-Carlo rejection method and estimate that $\sim 60$ AGNs would be sufficient to verify the existence of an $R-S_{\text {softex }}$ correlation of comparable strengths to those found in our original simulations (Figures 3 and 5). This would amount to an easily achievable $X M M-N u S T A R$ campaign of $\sim 600 \mathrm{ks}$.

It will be easier to narrow down the mechanism responsible for producing the soft excess in any given source if it is first fit with the simplest possible "observer's model" outlined here to locate it on the $R-S_{\text {softex }}$ plot. Additionally, the regions of this plot occupied by large samples of AGNs will also provide an indication of the most likely soft excess production mechanisms in the AGN population as a whole. The general simulation methodology adopted here also has much potential for distinguishing between competing models in other areas of both AGN science and other fields of astrophysics. 
We thank the anonymous referee for useful suggestions which improved the paper. C.S.R. thanks NASA for support under grant NNX12AE13G. We thank Jeremy Sanders for help with the use of his Veusz plotting package and Cole Miller for helpful discussions on Monte-Carlo simulation techniques.

\section{REFERENCES}

Alston, W. N., Vaughan, S., \& Uttley, P. 2013, MNRAS, 435, 1511

Arnaud, K. A. 1996, in ASP Conf. Ser. 101, Astronomical Data Analysis Software and Systems V, ed. G. H. Jacoby \& J. Barnes (San Francisco, CA: ASP), 17

Arnaud, K. A., Branduardi-Raymont, G., Culhane, J. L., et al. 1985, MNRAS, 217, 105

Ballantyne, D. R., McDuffie, J. R., \& Rusin, J. S. 2011, ApJ, 734, 112

Cerruti, M., Ponti, G., Boisson, C., et al. 2011, A\&A, 535, A113

Chevallier, L., Collin, S., Dumont, A.-M., et al. 2006, A\&A, 449, 493

Corral, A., Della Ceca, R., Caccianiga, A., et al. 2011, A\&A, 530, A42

Crummy, J., Fabian, A. C., Gallo, L., \& Ross, R. R. 2006, MNRAS, 365, 1067

D’Ammando, F., Bianchi, S., Jiménez-Bailón, E., \& Matt, G. 2008, A\&A, 482, 499

Dauser, T., Svoboda, J., Schartel, N., et al. 2012, MNRAS, 422, 1914

De Marco, B., Ponti, G., Cappi, M., et al. 2013, MNRAS, 431, 2441

Done, C., Davis, S. W., Jin, C., Blaes, O., \& Ward, M. 2012, MNRAS, 420,1848

Emmanoulopoulos, D., McHardy, I. M., \& Papadakis, I. E. 2011, MNRAS, 416, L94

Fabian, A. C., Kara, E., Walton, D. J., et al. 2013, MNRAS, 429, 2917

Fabian, A. C., Zoghbi, A., Ross, R. R., et al. 2009, Natur, 459, 540

Fabian, A. C., Zoghbi, A., Wilkins, D., et al. 2012, MNRAS, 419, 116

Gierliński, M., \& Done, C. 2004, MNRAS, 349, L7

Harrison, F. A., Craig, W. W., Christensen, F. E., et al. 2013, ApJ, 770, 103

Holt, S. S., Mushotzky, R. F., Boldt, E. A., et al. 1980, ApJL, 241, L13

Jin, C., Ward, M., Done, C., \& Gelbord, J. 2012, MNRAS, 420, 1825

Kara, E., Fabian, A. C., Cackett, E. M., Miniutti, G., \& Uttley, P. 2013, MNRAS, 430, 1408
Laha, S., Dewangan, G. C., Chakravorty, S., \& Kembhavi, A. K. 2013, ApJ, 777,2

Magdziarz, P., \& Zdziarski, A. A. 1995, MNRAS, 273, 837

Matt, G., Marinucci, A., Guainazzi, M., et al. 2014, arXiv:1401.5235

Middleton, M., Done, C., \& Gierliński, M. 2007, MNRAS, 381, 1426

Miller, L., \& Turner, T. J. 2013, ApJL, 773, L5

Miniutti, G., Ponti, G., Greene, J. E., et al. 2009, MNRAS, 394, 443

Nandra, K., O’Neill, P. M., George, I. M., \& Reeves, J. N. 2007, MNRAS, 382, 194

Page, K. L., Schartel, N., Turner, M. J. L., \& O'Brien, P. T. 2004, MNRAS, 352,523

Paul, B. 2013, IJMPD, 22, 41009

Ponti, G., Gallo, L. C., Fabian, A. C., et al. 2010, MNRAS, 406, 2591

Ponti, G., Miniutti, G., Cappi, M., et al. 2006, MNRAS, 368, 903

Pravdo, S. H., Nugent, J. J., Nousek, J. A., et al. 1981, ApJ, 251, 501

Reynolds, C. S., \& Fabian, A. C. 2008, ApJ, 675, 1048

Risaliti, G., Harrison, F. A., Madsen, K. K., et al. 2013, Natur, 494, 449

Risaliti, G., Nardini, E., Salvati, M., et al. 2011, MNRAS, 410, 1027

Rivers, E., Markowitz, A., Duro, R., \& Rothschild, R. 2012, ApJ, 759, 63

Rivers, E., Markowitz, A., \& Rothschild, R. 2013, ApJ, 772, 114

Ross, R. R., \& Fabian, A. C. 2005, MNRAS, 358, 211

Saez, C., Chartas, G., Brandt, W. N., et al. 2008, AJ, 135, 1505

Schurch, N. J., \& Done, C. 2008, MNRAS, 386, L1

Singh, K. P., Garmire, G. P., \& Nousek, J. 1985, ApJ, 297, 633

Takahashi, T., Mitsuda, K., Kelley, R., et al. 2012, Proc. SPIE, 8443, $84431 \mathrm{Z}$

Tatum, M. M., Turner, T. J., Miller, L., \& Reeves, J. N. 2013, ApJ, 762, 80

Turner, T. J., Miller, L., Kraemer, S. B., Reeves, J. N., \& Pounds, K. A. 2009, ApJ, 698, 99

Turner, T. J., Miller, L., Reeves, J. N., \& Kraemer, S. B. 2007, A\&A, 475,121

Vasudevan, R. V., Brandt, W. N., Mushotzky, R. F., et al. 2013, ApJ, 763,111

Walton, D. J., Nardini, E., Fabian, A. C., Gallo, L. C., \& Reis, R. C. 2013, MNRAS, 428, 2901

Walton, D. J., Reis, R. C., \& Fabian, A. C. 2010, MNRAS, 408, 601

Winter, L. M., Danforth, C., Vasudevan, R., et al. 2011, ApJ, 728, 28

Zhong, X., \& Wang, J. 2013, ApJ, 773, 23

Zoghbi, A., Fabian, A. C., \& Gallo, L. C. 2008, MNRAS, 391, 2003

Zoghbi, A., Fabian, A. C., Uttley, P., et al. 2010, MNRAS, 401, 2419 